\title{
Trio : un facteur d'échange des GTPases Rho aux multiples facettes impliqué dans le guidage axonal
}

\author{
Les facteurs d'échanges nucléotidiques (GEF) des GTPases \\ Rho remodèlent le cytosqu elette d'actine en activant leurs \\ cibles, les GTPases R ho, et sont ainsi impliqués dans de \\ nombreux processus physiologiques. Trio est le premier \\ membre d'une famille de protén es multifonctionnelles \\ possédant deux domaines fonctionnels d'échange dont la \\ spécificité est différente, le premier activant $R$ ac et le \\ second R hoA. Trio possède aussi de nombreux domaines
}

\begin{abstract}
d'interaction protéineprotéine, et semble donc se situ er à un carrefour de différentes voies de signalisation. La liaison de Trio avec des proténes qui lient l'actine, suggère en ou tre que l'activation des GT Pases par Trio est contrôlée par sa localisation spécifique près du cytosquelette. De plus, l'identification d'orthologues de Trio chez les invertébrés a permis de proposer un e fonction essen tielle de Trio dans le guidage axonal.
\end{abstract}

L es GTPases de la famille Rho contrôlent l'organisation du cytosquelette d'actine [1,2], qui joue un rôle capital dans une variété de processus physiologiques tels que la prolifération, la motilité, la morphologie et la différenciation cellulaires. L'effet sur le cytosquelette d'actine des GTPases Rho les plus étudiées a été très bien caractérisé dans les fibroblastes: Cdc42 induit la formation de structures riches en actine appelées les filopodes, Rac promeut la formation de replis membranaires appelés ruffles, et RhoA la formation de fibres de tension [1]. RhoG active indépendamment les GTPases Rac et Cdc42 [3]. L'activation de Rac et de Cdc42 est associée à la motilité cellulaire dans de nombreux systèmes, tandis que l'activité de RhoA est associée à l'adhérence à la matrice extracellulaire, indiquant que les voies de signalisation $\mathrm{Rac} / \mathrm{Cdc} 42$ et RhoA sont en général antagonistes.

La fonction des GTPases dépend de l'équilibre entre leurs formes inactive, liée au GDP, et active, liée au GTP. Sous forme GTP, les GTPases interagissent avec des protéines effectrices spécifiques dont l'activation permet les modifications du cytos1316 entre les formes inactive et active des GTPases est régi par deux types de protéines: les facteurs d'échanges nucléotidiques ou GEF ( pour guanine nucleotide exchange factors) qui activent les GTPases en accélérant l'échange du GDP pour le GTP, et les GTPase activating proteins (GAP) qui assurent le retour de la GTPase à l'état inactif par la stimulation de I'hydrolyse du GTP [5]. Les protéines GEF qui activent les GTPases Rho, appelées RhoGEF, appartiennent à une famille caractérisée par la présence d'un domaine DH (pour Dbl-homology domain, en référence à l'oncogène Dbl, un des premiers Rho-GEF identifié) qui porte l'activité catalytique d'échange [6]. Au domaine $\mathrm{DH}$ de ces GEF est toujours associé un domaine $\mathrm{PH}$ (Pleckstrin-homology domain) dont le rôle le mieux établi est le ciblage des protéines à la membrane plasmique grâce à sa liaison aux phospholipides [7]. Les domaines PH participent également à des interactions protéine-protéine $[8,9]$ et peuvent aussi moduler l'activité catalytique du domaine DH adjacent [10, 11]. L'association systématique d'un domaine $\mathrm{DH}$ avec un domaine $\mathrm{PH}$ suggère que ce module constitue l'unité fonctionnelle minimale pour I'activation des GTPases Rho in vivo.
En plus du module DH-PH, les RhoGEF contiennent aussi de nombreux domaines accessoires d'interaction protéine-protéine, ce qui laisse penser que I'activation des GTPases par les Rho-GEFs peut être contrôlée par différentes voies de signalisation. Ce contrôle participe probablement à la spécificité de la réponse biologique induite par chaque RhoGEF.

\section{La protéine multifonctionnelle Trio}

La protéine humaine Trio de $330 \mathrm{kDa}$ a été isolée au cours d'un crible double-hybride en utilisant comme appât la tyrosine phosphatase transmembranaire LAR (leucocyte common antigen related) [12], un homologue de la tyrosine phosphatase spécifique du lymphocyte T CD45. Si la fonction de LAR chez les mammifères est encore mal comprise, son orthologue chez la drosophile semble en revanche impliqué dans le guidage axonal de certains moto neurones [13].

Trio est une protéine ubiquitaire dont l'organisation structurale est relativement complexe (figure 1) [14]. En effet, elle possède 3 domaines enzymatiques: deux domaines GEF (GEF1 et GEF2) et un domaine 


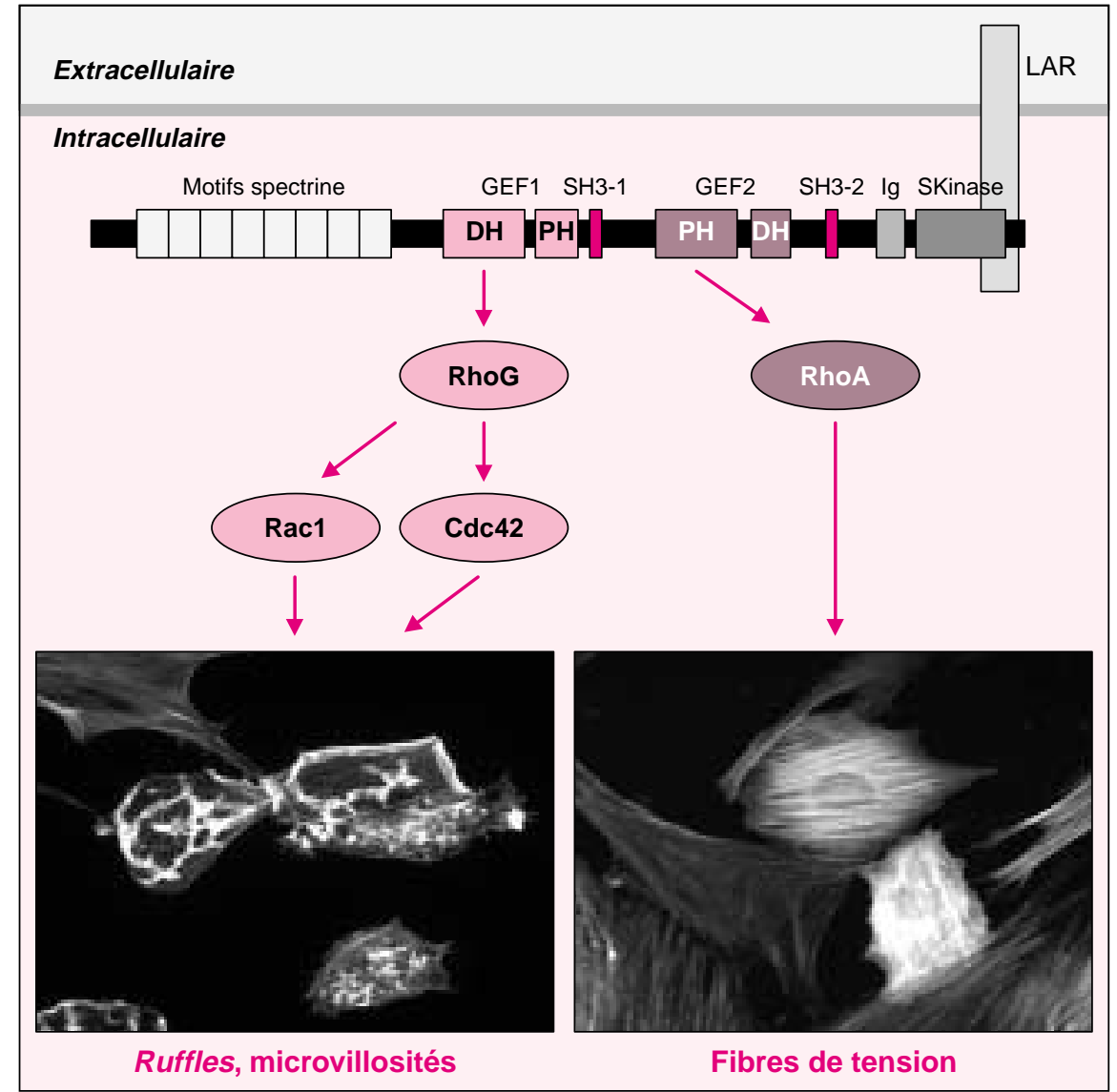

Figure 1. Trio est une protéine multifonctionnelle qui contrôle l'activation de plusieurs GTPases in vivo. Trio est le premier membre d'une nouvelle famille de protéines multifonctionnelles. Elle possède en effet deux domaines GEF (GEF1 et GEF2) de spécificité différente, chacun composé d'un domaine DH associé à un domaine PH. GEF1 active la voie de signalisation Rac/Cdc42 via I'activation directe de la GTPase RhoG, ce qui permet la formation de replis membranaires (ruffles) et de microvillosités. GEF2 active la GTPase RhoA impliquée dans la formation des fibres de tension. Trio possède en outre plusieurs domaines d'interaction protéine-protéine comme les motifs spectrine, les motifs SH3, Immunoglobuline-like et un domaine kinase. Ces deux derniers, Ig-like et kinase, sont responsables de l'interaction de Trio avec la protéine phosphatase membranaire LAR. GEF: guanine nucleotide exchange factor; DH: Dbl-homology domain; PH: Pleckstrin-homology domain; LAR: leucocyte common antigen related.

kinase fortement homologue à celui de la famille des sérine kinases dépendantes de la calmoduline. Trio possède en outre des domaines d'interaction protéine-protéine tels que des motifs $\mathrm{SH} 3$, immunoglobuline $(\mathrm{lg})$-like et des motifs répétés trouvés dans la spectrine, suggérant que Trio puisse être à un carrefour de différents réseaux de signalisation (figure 1). Ce sont les domaines Ig et kinase de Trio qui sont responsables de l'interaction avec la phosphatase LAR [14]. Ainsi, la liaison de LAR à $\mathrm{m} / \mathrm{s} \mathrm{n}^{\circ} 12$, vol. 17, décembre 2001 que $\operatorname{Rac}[14,15]$. L'expression du domaine GEF1 de Trio dans des fibroblastes provoque la formation de replis membranaires et de microvillosités, effets morphologiques révélateurs de l'activation concommitante de Rac et de Cdc42 par RhoG (figure 1). La GTPase RhoG semble être effectivement la cible directe du domaine GEF1 in vivo puisque le remodelage du cytosquelette induit par ce domaine est complètement bloqué par des formes dominantes négatives de la GTPase et de ses effecteurs spécifiques [15].

En revanche, l'expression dans les fibroblastes du domaine GEF2 de Trio promeut la formation de fibres de tension, qui est un événement dépendant de RhoA [16] (figure 1). Ceci est tout à fait cohérent avec le fait que ce domaine stimule spécifiquement I'échange GDP/GTP de RhoA in vitro [14]. Trio est ainsi le premier membre d'une nouvelle famille de protéines complexes possédant deux domaines GEF fonctionnels et de spécificité différente, pouvant relier la signalisation de plusieurs GTPases in vivo [16]. D'autres protéines possédant des caractéristiques structurales similaires ont été isolées depuis chez I'homme, le rat, la drosophile et C. elegans (figure 2).

Cependant, il reste encore à déterminer si les deux domaines GEF de Trio sont actifs simultanément in vivo, puisque seul le premier semble jouer un rôle majeur dans la fonction de Trio. Ceci est d'autant plus important que ces deux domaines stimulent des voies de signalisation antagonistes.

\section{Les partenaires de Trio}

Trio permettrait à la phosphatase d'avoir accès au contrôle du cytosquelette d'actine en modulant I'activité des GTPases Rho.

\section{Trio contrôle l'activation de plusieurs GT Pases}

Le domaine GEF1 de Trio est capable d'accélérer spécifiquement I'échange GDP/GTP sur la GTPase Racl in vitro, mais surtout sur la GTPase RhoG, qui est un bien meilleur substrat pour ce domaine
L'organisation structurale de Trio suggère que cette protéine multifonctionnelle participe à des complexes multiprotéiques et puisse ainsi intégrer différents signaux lui permettant de contrôler finement l'activation des GTPases Rho. Plusieurs données expérimentales étayent cette hypothèse: deux cribles doublehybride effectués de façon indépendante en utilisant comme appât le premier domaine GEF de Trio ont permis d'isoler deux protéines qui 


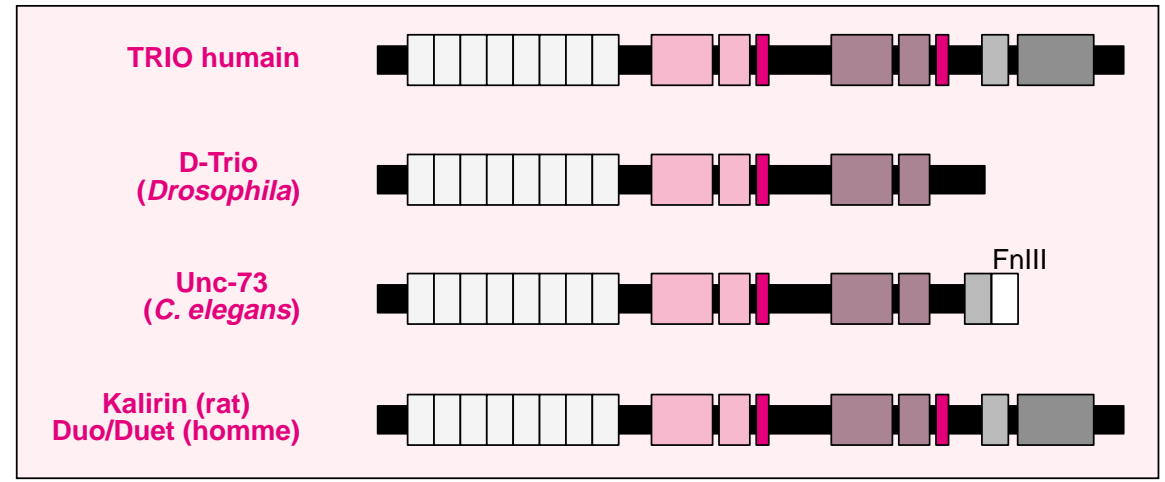

Figure 2. La famille des protéines Trio. Depuis la caractérisation de la protéine Trio humaine, des orthologues de Trio ont été identifiés chez le nématode $\mathrm{C}$. elegans et la drosophile. Les membres de cette famille contiennent tous deux domaines GEF activant les GTPases Rho. La séquence entre ces différents orthologues diverge uniquement dans la partie $\mathrm{C}$-terminale, après le domaine GEF2. unc-73 se termine par un domaine Fibronectine de type III (FnIII). Chez les mammifères, d'autres protéines possédant le même type d'organisation structurale existent, telles que la kalirine chez le rat, et les protéines Duo et Duet chez l'homme.

lient l'actine. La première est la protéine Tara qui semble avoir une activité de stabilisation du cytosquelette d'actine [17] (figure 3). A l'heure actuelle, peu de choses sont connues sur la signification physiologique de I'interaction entre Tara et Trio. La seconde protéine identifiée lie spécifiquement le domaine $\mathrm{PH} 1$ de Trio: c'est la filamine, qui réticule l'actine grâce à sa capacité d'homodimérisation [9] (figure 3). La filamine interagit avec de nombreuses protéines transmembranaires dont les intégrines, et permet ainsi de relier la membrane plasmique au cytosquelette d'actine [18]. De nombreux travaux montrent que la filamine est absolument essentielle pour la motilité cellulaire aussi bien en culture qu'in vivo lors de la migration des neurones corticaux au cours du développement du cerveau humain [19, 20]. L'importance fonctionnelle de I'interaction Trio/filamine a été démontrée en utilisant une lignée de mélanomes qui n'expriment plus la filamine. Dans ces cellules, le domaine GEF1 de Trio n'induit plus le remodelage du cytosquelette d'actine, alors qu'il en est parfaitement capable dans les mêmes cellules exprimant stablement la fila- n'affecte en aucune façon l'activité d'échange in vitro de ce domaine GEF1, ces résultats suggèrent que la filamine est une protéine d'ancrage au cytosquelette, où Trio viendrait se fixer via son domaine $\mathrm{PH} 1$ pour activer la cascade de signalisation de Rac.

Par ailleurs, le lien entre les GTPases Rho et Trio est encore renforcé par la mise en évidence d'une interaction directe entre le domaine Ig-like de Trio et la GTPase RhoA, permettant probablement d'amplifier le signal activé par le domaine GEF2 [21] (figure 3). De façon plus générale, I'activation des GTPases Rho par Trio semble donc être finement contrôlée par la localisation spécifique de Trio, localisation qui est probalement dictée par son interaction avec ses différents partenaires associés au cytosquelette.

\section{Finalement quelle est la fonction biologique de Trio ?}

Le rôle exact de la protéine Trio chez les mammifères est encore mal compris, mais l'analyse de différents modèles génétiques chez les invertébrés permet d'envisager une fonction essentielle de Trio dans la physiologie neuronale chez l'adulte et au

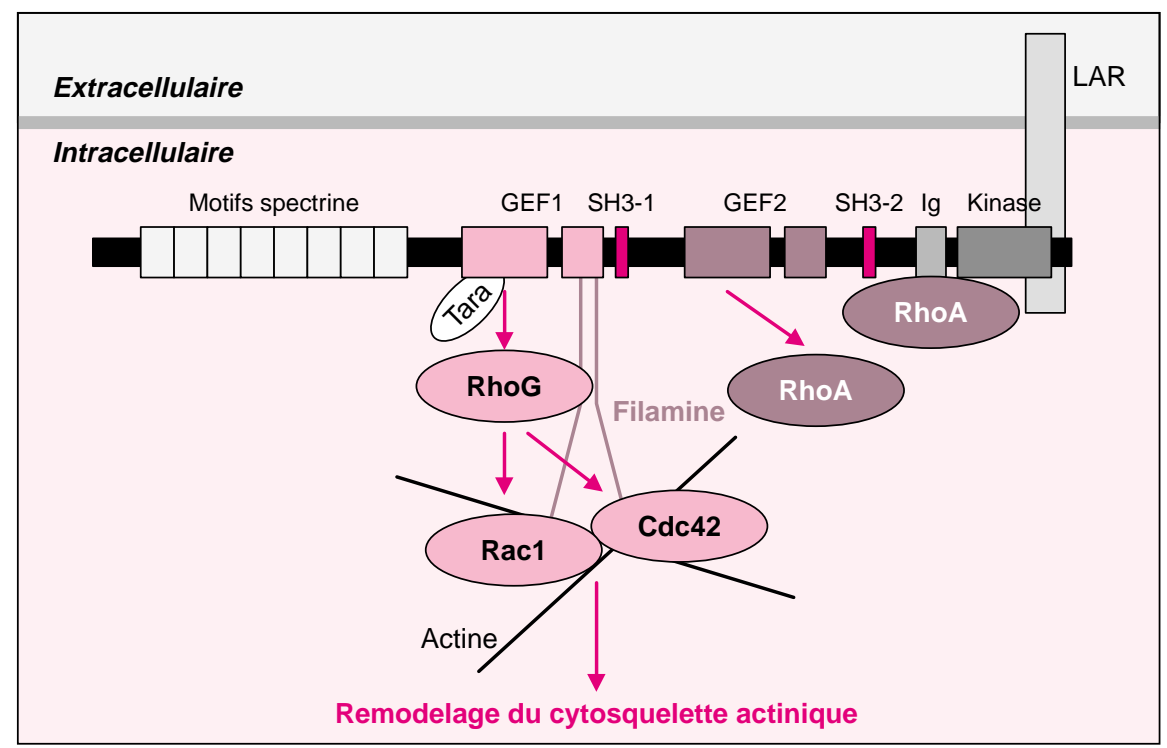

Figure 3. Les partenaires de Trio. Trio participe à des complexes multiprotéiques associés au cytosquelette actinique. En utilisant différentes approches, il a été montré que Trio se lie, par son domaine GEF1, à des protéines liant l'actine telles que la filamine et une nouvelle protéine Tara. Ces interactions semblent jouer un rôle dans le remodelage du cytosquelette d'actine. Trio interagit également, par son domaine lg, avec la GTPase RhoA ce qui permet probablement d'amplifier le signal activé par le domaine GEF2. 
cours du développement, en particulier dans le guidage axonal. Dans ce processus, la croissance des axones vers leur cible appropriée est déterminée par la détection des molécules de guidage par la structure spécialisée présente au bout de l'axone, le cône de croissance. Les cônes de croissance répondent aux signaux attractifs par la production de structures actiniques, dont la formation est dépendante de l'activité des GT Pases Rho [22].

L'analyse du mutant unc-73 (pour uncoordinated) chez le nématode $C$. elegans révèle des mutations dans un gène dont l'organisation structurale ressemble fortement à Trio, sauf dans sa partie C-terminale où un domaine fibronectine de type III (FnIII) remplace le domaine kinase (figure 2). Les mutants unc-73 présentent de nombreux défauts de migration des cellules neuronales, mais aussi de guidage et de fasciculation des axones [23].

Plusieurs études indépendantes ont identifié l'orthologue de Trio chez la drosophile [24-27]. Drosophila Trio (D-Trio) a été en particulier isolé par un crible permettant d'identifier des gènes impliqués dans le guidage axonal des cellules photoréceptrices de I'œil [24]. D-Trio possède une forte homologie avec la protéine Trio humaine, mais se termine après le deuxième domaine $\mathrm{PH}$ et ne possède donc pas le domaine kinase (figure 2). L'activité du domaine GEF1 de D-Trio, par sa capacité à activer Rac, est essentielle pour une navigation correcte des axones dans ce système, alors que l'activité du GEF2 n'est pas requise. Ceci indique que le premier domaine GEF de DTrio a un rôle majeur dans le guidage axonal en stimulant la voie de signalisation de Rac, ce qui avait été également observé dans le cas de unc-73. De plus, D-Trio interagit génétiquement avec d'autres gènes dont les produits ont été impliqués dans le guidage axonal, tels que la kinase effectrice de Rac, PAK (p21activated kinase), et I'adaptateur moléculaire Dock [24] (figure 4). Sachant que PAK est recrutée à la membrane plasmique par sa liaison avec Dock, il est tentant de proposer qu'une fois PAK recrutée à la mem-

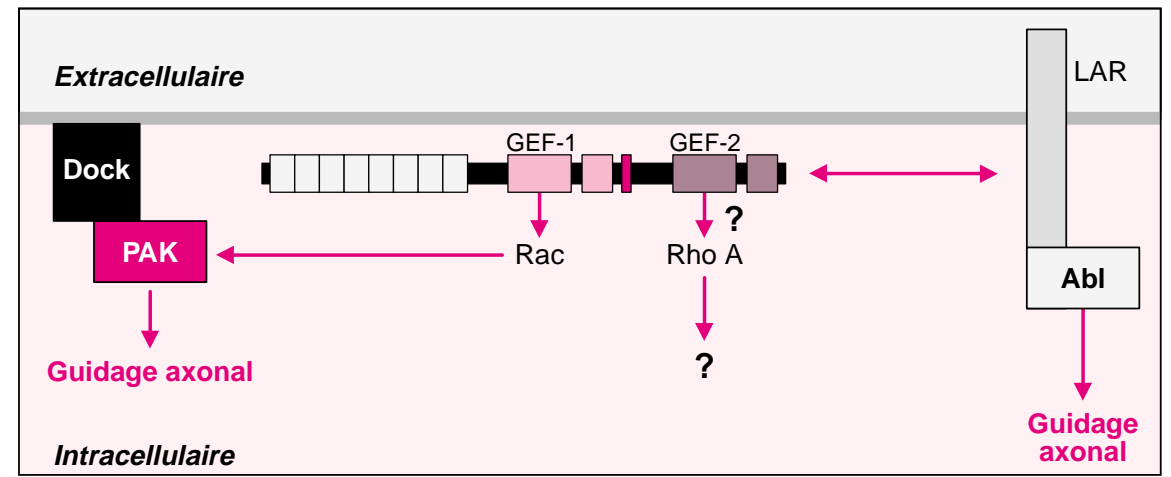

Figure 4. Fonction de Trio dans le guidage axonal chez la drosophile. L'analyse des données génétiques chez $C$. elegans et la drosophile a permis d'envisager une fonction essentielle des orthologues de Trio dans le guidage axonal. Ce processus requiert uniquement l'activité du GEF1. D-Trio interagit génétiquement avec la kinase effectrice de Rac, PAK, et l'adaptateur moléculaire Dock pour assurer un guidage axonal correct. Le modèle le plus probable est qu'une fois PAK recrutée à la membrane par Dock, elle soit activée par Trio via Rac. II semble de plus exister une coopération entre la voie Trio/Rac/PAK et celle impliquant LAR et son substrat Abl, qui sont aussi des acteurs du guidage axonal chez la drosophile. La fonction du GEF2 reste encore obscure, mais des données récentes suggèrent que celui-ci pourrait être impliqué dans la morphologie dendritique, via l'activation de RhoA.

brane plasmique, elle soit activée par D-Trio via Rac.

Si le rôle du premier domaine GEF de Trio semble être prépondérant dans le contrôle du guidage axonal par D-Trio, celui du second domaine reste obscur. Cependant, dans les mutants trio chez la drosophile, on observe chez l'adulte une extension anormale des dendrites au niveau d'une structure particulière du cerveau, les corps pédonculés, et ce phénotype est tout à fait similaire à celui observé dans des mutants de la GTPase RhoA [27]. Ainsi, D-Trio activerait $R a c$ via son premier domaine GEF pour contrôler le guidage axonal, et activerait RhoA via son second domaine pour contrôler la morphologie dendritique.

Sachant que Trio a été isolé grâce à son interaction avec la tyrosine phosphatase LAR, il est intéressant de noter que les mutants de l'orthologue de LAR chez la drosophile, dlar, présentent des défauts identiques à ceux de D-Trio au niveau de l'extension des moto-neurones et que ces deux protéines pourraient participer à la même voie de signalisation $[13,26,28]$. D'autre part, DTrio a été également identifié au cours d'un crible génétique visant à isoler des gènes participant à la voie de transduction de la tyrosine kinase Abl, qui est un substrat de la tyrosine phosphatase DLAR [25]. Toutes ces données suggèrent que D-Trio, via I'activité de son GEF1 ou GEF2, puisse faire le lien entre le complexe $D L A R / A B L$ et la voie de signalisation Rac (figure 4). Cependant, D-Trio ne possèdant pas le domaine kinase qui est responsable de l'interaction LAR/ T rio chez les mammifères, il est possible que D-Trio et DLAR participent à des voies de signalisation communes aboutissant au contrôle du guidage axonal, mais sans interaction directe entre les deux protéines.

Plus récemment, le gène codant pour Trio a été invalidé chez la souris [29]. Les embryons trio-1- meurent au cours de l'embryogenèse tardive entre le stade embryonnaire 15.5 et la naissance. L'analyse des embryons montre un défaut de la myogénèse, puisque certaines myofibrilles des muscles squelettiques présentent une structure sphérique inhabituelle. De plus, la vague de différenciation musculaire correspondant à la myogenèse secondaire ne semble pas se produire chez les embryons trio-1Peu d'informations sont connues à l'heure actuelle sur le rôle exact de 
Trio dans la différenciation musculaire, mais le rôle présomptif de Trio dans ce processus est cohérent avec l'observation selon laquelle les GTPases de type Rho semblent jouer un rôle crucial dans ce phénomène. II reste toutefois à montrer que le défaut présenté par les embryons trio- ${ }^{-1-}$ est bien dû à une altération de l'activation des GTPases cibles de Trio.

La perte de l'expression de Trio provoque également des anomalies du développement du cerveau, mais celles-ci sont relativement peu sévères compte tenu des résultats obtenus chez les invertébrés. En effet, on note une désorganisation de la région de I'hippocampe et du bulbe olfactif, mais l'organisation globale du cerveau de ces embryons est peu affectée.

Une hypothèse possible pour expliquer le faible effet de l'absence de Trio sur le développement cérébral est l'existence d'une redondance fonctionnelle avec des protéines de la même famille. En effet, chez les mammifères, la kalirine chez le rat et son orthologue Duo/ Duet chez I'homme ont une organisation structurale très similaire à celle de Trio [30-32] (figure 2). L'expression de la kalirine est restreinte au cerveau adulte, et une étude récente a montré qu'elle serait impliquée dans le remodelage du cytosquelette d'actine dans les dendrites de neurones corticaux, réglant ainsi leur morphologie [33]. Au vu de ces données, on peut envisager que le phénotype relativement atténué observé dans le cerveau des embryons trio-1- soit dû à une redondance fonctionnelle avec la kalirine.

\section{Conclusions}

Trio est donc une molécule tout à fait intéressante par sa capacité à contrôler l'activité des GTPases Rho sur le cytosquelette d'actine. Elle semble contrôler très finement l'activation des GTPases grâce à sa liaison à ses partenaires du cytosquelette. Pour l'instant, seul le premier domaine GEF semble jouer un rôle majeur dans la fonction de Trio, alors que la fonction du second
Sachant que les GT Pases cibles de ces deux domaines GEF activent des voies de signalisation antagonistes, il est probable qu'ils ne sont pas actifs en même temps. En outre, l'activité des domaines GEF de Trio est probablement réglée par la fonction des nombreux autres domaines de Trio, comme le domaine kinase et les motifs SH 3. On peut suspecter qu'ils permettent à Trio d'intégrer d'autres signaux permettant de moduler I'activation des GT Pases Rho

\section{RÉFÉRENCES}

1. Hall A. Rho GTPases and the actin cytoskeleton. Science 1998; 279: 509-14.

2. Ory S, Jurdic P. Microtubules, compartiments cellulaires et GTPases Rho: ménage à trois pour une migration efficace. M ed Sci 2001; 17: 878-85.

3. Gauthier-Rouviere C, Vignal E, Meriane $M$, Roux P, M ontcourier P, Fort P. RhoG GTPase controls a pathway that independently activates Racl and Cdc42H s. M ol Biol Cell 1998; 9: 1379-94.

4. Van Aelst L, D'Souza-Schorey C. Rho GTPases and signaling networks. G enes Dev 1997; 11: 2295-322.

5. Boguski MS, McCormick F. Proteins regulating Ras and its relatives. $N$ ature 1993; 366: 643-54.

6. Stam JC, Collard JG. The DH protein family, exchange factors for Rho-like GTPases. Prog M ol Subcell Biol 1999; 22: 5183.

7. Lemmon MA, Ferguson KM. Signaldependent membrane targeting by pleckstrin homology (PH) domains. Biochem J 2000; 350: 1-18.

8. Yao L, Janmey $P$, Frigeri $L G$, et al. Pleckstrin homology domains interact with filamentous actin. J Biol Chem 1999; 274: 19752-61.

9. Bellanger JM, Astier C, Sardet C, O hta Y, Stossel TP, Debant A. The Racl- and RhoGspecific GÉF domain of Trio targets filamin to remodel cytoskeletal actin. Nat Cell Biol $2000 ; 2$ : 888-92.

10. Han J, Luby-Phelps K, Das B, \& al. Role of substrates and products of PI 3-kinase in regulating activation of Rac-related guanosine triphosphatases by Vav. Science 1998; 279: 558-60.
11. Nimnual AS, Yatsula BA, Bar-Sagi D. Coupling of Ras and Rac guanosine triphosphatases through the Ras exchanger Sos. Science 1998; 279: 560-3.

12. Streuli $M$, Krueger $N, X$, hall $L R$, Schlossman SF, Saito H. A new member of the immunoglobulin superfamily that has a cytoplasmic region homologous to the leucocyte common antigen. J Exp M ed 1988; 168: 1523-30.

13. Krueger NX, Van Vactor D, Wan HI, Gelbart WM, Goodman CS, Saito H. The transmembrane tyrosine phosphatase DLAR controls motor axon guidance in Drosophila. Cell 1996; 84: 611-22.

14. Debant A, Serra-Pages C, Seipel K, et al. The multidomain protein Trio binds the LAR transmembrane tyrosine phosphatase, contains a protein kinase domain, and has separate rac- specific and rho-specific guanine nucleotide exchange factor domains. Proc Natl A cad Sci USA 1996; 93: 5466-71.

15. Blangy A, Vignal E, Schmidt S, Debant $A$, Gauthier-Rouvière CPF. TriogEF1 controls Rac- and Cdc42-dependent cell structures through direct activation of RhoG. J Cell Sci 2000; 113: 729-39.

16. Bellanger JM, Lazaro JB, Diriong S, Fernandez $A, L a m b N$, Debant $A$. The two guanine nucleotide exchange factor domains of Trio link the Racl and the RhoA pathways in vivo. Oncogene 1998; 16: 147-52.

17. Seipel K, O'Brien SP, Iannotti E, Medley QG, Streuli $M$. Tara, a novel $F$-actin binding protein associates with the Trio guanine nucleotide exchange factor and regulates actin cytoskeletal organization. J Cell Sci 2001 ; 114: 389-99.

18. Stossel TP, Condeelis J, Cooley L, et al. Filamins as integrators of cell mechanics and signalling. $\mathrm{N}$ at Rev M ol Cell Biol 2001; 2: 138-45.

19. Cunningham $C C$, Gorlin JB, Kwiatkowski DJ, et al. Actin-binding protein requirement for cortical stability and efficient locomotion. Science 1992; 255: 325-7.

20. Fox JW, Lamperti ED, Eksioglu YZ, et al. Mutations in filamin 1 prevent migration of cerebral cortical neurons in human periventricular heterotopia. Neuron 1998; 21 : 1315-25.

21. M edley Q G, Serra-Pages C, I annotti E, et al. The Trio guanine nucleotide exchange factor is a rhoA target: binding of rhoA to the Trio immunoglobulin-like domain. J Biol Chem 2000; 275: 36116-23.

22. Luo L. Rho-GTPases in neuronal morphogenesis. Nat Rev Neurosci 2000; 1: 17380.

23. Steven $\mathrm{R}$, Kubiseski $\mathrm{TJ}$, Zheng $\mathrm{H}$, et al. UNC-73 activates the Rac GTPase and is required for cell and growth cone migrations in C. elegans. Cell 1998; 92: 785-95. 


\section{RÉFÉRENCES}

24. Newsome TP, Schmidt S, Dietzl G, et al. Trio combines with dock to regulate Pak activity during photoreceptor axon pathfinding in Drosophila. Cell 2000; 101: 283-94.

25. Liebl EC, Forsthoefel DJ, Franco LS, et al. Dosage-sensitive, reciprocal genetic interactions between the Abl tyrosine kinase and the putative GEF trio reveal trio's role in axon pathfinding. Neuron 2000; 26 : 10718.

26. Bateman J, Shu H, Van Vactor D. The guanine nucleotide exchange factor trio mediates axonal development in the Drosophila embryo. Neuron 2000; 26: 93-106.

27. Awasaki T, Saito M, Sone M, et al. The Drosophila trio plays an essential role in patterning of axons by regulating their directional extension. Neuron 2000; 26: 119-31.

28. Wills Z, Bateman J, Korey CA, Comer A, Van Vactor D. The tyrosine kinase Abl and its substrate enabled collaborate with the receptor phosphatase Dlar to control motor axon guidance. Neuron 1999; 22 : 301-12.
29. O'Brien SP, Seipel K, Medley QG, Bronson R, Segal $R$, Streuli M. Skeletal muscle deformity and neuronal disorder in Trio exchange factor-deficient mouse embryos. Proc Natl Acad Sci USA 2000; 97 : 12074-8.

30. Alam MR, Johnson RC, Darlington DN, $H$ and TA, Mains RE, Eipper BA. Kalirin, à cytosolic protein with spectrin-like and GDP/ GTP exchange factor-like domains that interacts with peptidylglycine alphaamidating monooxygenase, an integral membrane peptide-processing enzyme. J Biol Chem 1997; 272: 12667-75.

31. Colomer V, Engelender S, Sharp $A H_{\text {, }}$ et al. Huntingtin-associated protein 1 (HAP1) binds to a Trio-like polypeptide, with a racl guanine nucleotide exchange factor domain. Hum Mol Genet 1997; 6: 1519-25.

32. Kawai $T$, Sanjo $H$, Akira $S$. Duet is a novel serine/ threonine kinase with $\mathrm{Dbl}$ Homology (DH) and Pleckstrin-Homology $(\mathrm{PH})$ domains. Gene 1999; 227 : 24955

33. Penzes $P$, Johnson $R C$, Sattler $R$, et al. The neuronal Rho-GEF Kalirin-7 interacts with PDZ domain-containing proteins and regulates dendritic morphogenesis. Neuron $2001 ; 29: 229-42$.

\section{Jean-Michel Bellanger \\ Susanne Schmidt \\ Soline Estrach \\ Élodie Portales-C asamar \\ Sylvie Diriong \\ Anne Debant}

CRBM-Cnrs, 1919, route de M ende, 34293 M ontpellier, France.

\section{E-mail : mail debant@crbm.cnrs-mop.fr}

\section{Remerciements}

Les travaux du laboratoire sont soutenus majoritairement par la Ligue Nationale contre le Cancer (équipe labellisée) et par une subvention de I'Association de la Recherche contre le cancer.

\section{TIRÉS À PART}

A. Debant. 